Cahiers d'études africaines

\title{
Le centre Perevalnoe et la formation de militaires en Union soviétique
}

\section{Natalia Krylova}

Traducteur : Sonia Colpart

\section{OpenEdition \\ Journals}

Édition électronique

URL : http://journals.openedition.org/etudesafricaines/20728

DOI : $10.4000 /$ etudesafricaines.20728

ISSN : $1777-5353$

Éditeur

Éditions de l'EHESS

\section{Édition imprimée}

Date de publication : 1 juillet 2017

Pagination : 399-416

ISBN : 978-2-7132-2688-5

ISSN : 0008-0055

\section{Référence électronique}

Natalia Krylova, «Le centre Perevalnoe et la formation de militaires en Union soviétique », Cahiers d'études africaines [En ligne], 226 | 2017, mis en ligne le 01 avril 2019, consulté le 01 janvier 2020. URL : http://journals.openedition.org/etudesafricaines/20728; DOI : 10.4000/etudesafricaines.20728 


\section{Le centre Perevalnoe et la formation de militaires en Union soviétique}

L'histoire de la formation de militaires étrangers dans les établissements d'enseignement militaire soviétiques remonte à la Seconde Guerre mondiale, lorsque plusieurs d'entre eux prenaient en charge la préparation de spécialistes étrangers, en lien avec la formation d'unités combattantes étrangères pour lutter contre le fascisme. Des militaires roumains, polonais, tchécoslovaques, yougoslaves, étaient instruits sur le territoire de l'uRss. Plusieurs grandes unités, parmi lesquelles deux armées polonaises, un corps d'armée tchécoslovaque, deux divisions roumaines, des brigades d'infanterie et de blindés yougoslaves, ainsi que des dizaines d'autres unités et subdivisions, furent organisées, formées et armées avec l'aide de l'Union soviétique (Shaïkin 2013 : 21). C'est à travers cette histoire, mais aussi dans la spécificité des relations entre l'Union soviétique et les pays du continent africain, que souhaite s'inscrire notre recherche à caractère exploratoire sur la formation des combattants de mouvements de libération et officiers africains dans les établissements d'enseignement militaire en URSS dans les années 1960-1990.

Dans la période à laquelle nous nous intéressons de façon prioritaire ici, et qui commence après la Conférence de Bandung des peuples afroasiatiques et la proclamation de l'indépendance du Ghana en 1958, l'Union soviétique a renforcé sa présence sur le continent africain, et s'est efforcée d'établir des relations diversifiées - diplomatiques, commerciales, sociales et culturelles - avec chacun des pays africains qui proclamait son indépendance. Lorsqu'à la fin des années 1950, des mouvements pour l'indépendance nationale sont apparus en Afrique subsaharienne, l'Union soviétique s'est placée du côté des dirigeants des mouvements qui, tout en étant engagés dans les luttes anticoloniales, cherchaient à promouvoir la construction d'une "société sans classe et sans propriété individuelle». De toute évidence, l'état des relations entre les deux grandes puissances de l'époque, à savoir entre l'URSS et les États-Unis, explique cette prise de position. Cependant, ces mouvements étaient considérés comme participant de la lutte antiimpérialiste et non simplement comme des volets de la confrontation entre Moscou et Washington (Shubin 2013: 11). C'est à ce titre que le Kremlin établit, au lendemain des Indépendances, des relations diplomatiques avec plusieurs États d'Afrique subsaharienne et conclut des accords d'amitié et de coopération avec plusieurs d'entre eux. 
Dès le milieu du $\mathrm{XX}^{\mathrm{e}}$ siècle, l'URSS commença à accorder aux jeunes États indépendants du continent africain une aide militaire et militaro-technique à grande échelle, dont les bénéficiaires principaux furent l'Angola, la Somalie et plus tard l'Éthiopie; d'autres pays africains comme le Congo, la GuinéeBissau, le Cap Vert, la Guinée, le Mozambique et le Mali ont aussi compté parmi les destinataires de cette aide qui incluait aussi bien l'armement et les équipements techniques que l'encadrement militaire. Sous bien des rapports, l'activité de l'URSS en Afrique était prédéterminée par la course aux armements dans le cadre de la Guerre froide. Il revenait cependant aux nouveaux leaders africains de résoudre la question de la recherche de partenaires dans le cadre de la Défense nationale. Certains pays africains firent le choix de l'URSS et de ses alliés, sans cependant nécessairement rompre les liens militaires avec les anciennes métropoles (Korendiasov 2015: 10).

En 1953, la préparation des militaires étrangers était assurée par 21 académies et instituts militaires, 27 écoles militaires, 30 cours de perfectionnement pour les officiers et par des écoles supérieures pour officiers, auxquels il faut ajouter 4 établissements d'enseignement supérieur de la Flotte de la marine militaire (Direction générale de la coopération militaire internationale 2001 : 118). De 1956 à 1959, Moscou accueillit des militaires égyptiens et guinéens qui commençaient à arriver pour étudier en URSS. Les différentes formations s'adressaient aussi bien aux États déjà indépendants qu'à ceux étant encore sous le joug colonial, aux États d'orientation socialiste comme à ceux, tel le Sénégal, dont les choix sociétaux étaient ouvertement libéraux.

Au cours des années 1960, le nombre de demandes pour étudier dans les établissements du ministère de la Défense (MD) de l'URSS augmenta ; ces demandes de formation portaient d'abord sur des préparations de courte durée. Afin de satisfaire aux besoins des États demandeurs, de nouveaux établissements d'enseignement supérieur furent alors créés, parmi lesquels l'École militaire d'infanterie à Odessa, l'École militaire de l'air et le Centre du personnel technique aéronautique des Forces militaires aériennes à Frounze, le Centre d'enseignement-165, Perevalnoe, en Crimée, les centres de préparation et de perfectionnement des opérateurs d'artillerie pour la défense aérienne et les équipages de bateaux de petites dimensions ainsi que les vaisseaux de la flotte militaire navale, etc. En raison du grand nombre de demandes, mais aussi pour pallier la disparité des niveaux de base des candidats, des méthodes de sélection spécifiques furent introduites. Elles comprenaient des missions des commissions spéciales du MD de l'URSS dans les pays concernés pour aider à la sélection des candidats afin de limiter le nombre de recalés pour raisons de santé ou de faible niveau de formation générale (ibid. : 120). D'une manière générale, le MD de l'URSS a conçu dans les années 1960-1980 un puissant système de centres de formation, d'établissements, d'académies, où les militaires étrangers ont pu recevoir une préparation professionnelle diversifiée (de la guerre de guérilla à la formation politicoidéologique et socio-psychologique) ${ }^{1}$.

1. Les centres d'études où les étudiants étrangers, y compris africains, faisaient leur préparation militaire étaient éparpillés dans le pays, et se trouvaient surtout à la 
Les sources, le contexte et les méthodes

Un point de méthodologie s'impose. Alors que les recherches sur la formation des étudiants africains dans les établissements d'enseignement supérieur soviétiques/russes se développent depuis une quinzaine d'années, notamment en Russie et en France, les recherches sur la formation des militaires africains sont encore très rares ${ }^{2}$, et il n'existe que très peu de publications, émanant le plus souvent de l'administration russe, sur cette question. L'éclatement de l'Union soviétique ayant disséminé plusieurs établissements d'études militaires en URSS qui se trouvent dans des pays désormais indépendants, il est de fait difficile d'accomplir un travail de terrain permettant de décrire et analyser l'activité de l'ensemble des centres d'étude soviétiques chargés de la préparation des militaires africains ; d'autant plus que l'accessibilité aux archives militaires soviétiques, après une période d'ouverture euphorique, a été réduite de manière drastique. Il n'en existe pas moins quelques sources accessibles, qui permettent de reconstituer la vie dans les centres de formation militaires, notamment à Perevalnoe ${ }^{3}$, et de croiser différents points de vue. En effet, des vétérans ont créé progressivement des unions ou associations, des portails internet, sites, forums, sur lesquels ils peuvent, depuis le début des années 1990, échanger des informations, évoquer des événements passés, mais aussi publier leurs expériences ou leurs souvenirs sous forme d'essais, de notes, de récits en prose ou en vers. Ce sont des plateformes

périphérie : en Sibérie, Extrême-Orient, Turkménistan, Azerbaïdjan, Kirghizistan, Kazakhstan. Pour un témoignage sur ces différents centres, voir celui d'A. Melnik traducteur militaire, diplômé de l'Institut militaire des langues étrangères -385 у.Учебные чентры для иностранчев в СССР (Z-85u. Les centres d'études pour étrangers en URSS). Сайт ВИИЯ (Военного института иностранных языков), site internet VIIJA (Institut militaire des langues étrangères), $<$ http://vkimo.com/node/860>.

2. Voir l'article récent sur les anciens militaires du Zimbabwe formés en URSS de J. AleXAnder \& J. A. MCGRegor (2017). V. Shubin, directeur adjoint de l'Institut d'Afrique à Moscou, a entrepris depuis plusieurs années d'importantes recherches sur l'histoire des relations internationales entre différents pays d'Afrique et l'URSS, notamment sur les luttes de libération en Afrique australe, et s'est fortement intéressé aux questions militaires, ce qui l'a parfois conduit à aborder rapidement les questions de la formation des militaires. La vie quotidienne dans les centres de formation militaire est parfois évoquée lors de recherches sur les étudiants africains en URSS. Ainsi, l'historien S. V. Mazov note des cas isolés de conflits au niveau de la vie quotidienne entre la jeunesse locale et les élèves guinéens qui étudiaient dans les Cours centraux de préparation et de perfectionnement des cadres de l'aviation du MD à Frounze. Â côté de certaines manifestations de « racisme ordinaire », il y avait, écrit-il, « des incompréhensions nées de l'ignorance de choses élémentaires se rapportant à la psychologie, aux coutumes, ou aux croyances des Africains » (MAZOV 1999: 92-93).

3. Quelques informations ont également été recueillies sur l'École supérieure militaropolitique toutes troupes de Minsk, et sur l'École d'infanterie de Riazan (devenue ensuite l'École militaire supérieure des troupes aéroportées de Riazan). 
originales d'information où s'entretient un esprit de corps sur fond de nostalgie pour l'époque soviétique, émaillée néanmoins de quelques critiques à propos de telle ou telle question professionnelle ou politico-idéologique. Tenus régulièrement à jour, ces sites informent sur la situation et le devenir des uns et des autres (décorations, ordres militaires), actualisent les listes de vétérans, annoncent leurs rencontres et mettent à jour la chronique nécrologique. Ainsi, sur le site de l'Union des vétérans de l'Angola ${ }^{4}$, figurent les rubriques suivantes : «Je recherche ceux qui ont servi avec moi », "Livre de mémoire », «Étapes sur notre route», ainsi qu'une galerie de photos, etc. D'anciens traducteurs militaires ayant travaillé dans les centres de formation et qui y occupaient une position stratégique entre militaires africains et encadrement soviétique ${ }^{5}$, ont publié leurs témoignages sur différents sites, tandis que d'autres ont répondu au début des années 2000 à des enquêtes de l'édition ukrainienne de la Komsomolskaïa Pravda (La Vérité des Jeunesses communistes) ${ }^{6}$. Ces témoignages sur leur expérience dans les centres de formation militaires pour partisans et officiers venus d'Afrique comptent parmi les premiers à être publiés en Russie. De par leur caractère relativement spontané, ces matériaux auto-documentaires constituent, en l'absence d'enquêtes approfondies, une source utile pour appréhender la formation dispensée aux Africains élèves de Perevalnoe ainsi que leur vie quotidienne ${ }^{7}$. Nous avons également pu nous appuyer sur les notes du ministère des Affaires étrangères de l'URSS, de la Direction générale de la coopération militaire internationale du ministère de la Défense, sur les enquêtes et commentaires, bien que sommaires, des services diplomatiques accrédités durant les années 1960-1990 dans quelques pays du continent africain, ainsi que sur les premiers entretiens que nous avons pu réaliser et sur les trop rares données statistiques que nous avons pu recueillir.

À cette première étape de la recherche, une place importante est réservée à la présentation descriptive de la formation dispensée dans les centres d'études militaires accueillant des partisans et/ou des futurs officiers africains, vue par les enseignants et traducteurs soviétiques, en particulier à Perevalnoe.

4. <www.veterangola.ru>.

5. Les traducteurs assuraient le travail des conseillers et des spécialistes. Très sollicité, ce groupe servait d'interface entre les élèves africains et l'encadrement militaire et était constitué avant tout par les élèves de l'Institut militaire des langues étrangères. Étaient aussi admis comme traducteurs et interprètes quelques diplômés des établissements d'enseignement civil destinés à devenir traducteursinterprètes ou professeurs de langues étrangères.

6. Organe du Komsomol de 1925 à 1991, la Komsomolskaïa Pravda est un quotidien populaire à grand tirage, un «tabloïde » qui mêle notamment des nouvelles "people » et des enquêtes assez sérieuses. Il existe différentes éditions, notamment pour le Belarus, la Moldavie et l'Ukraine. Cette dernière a, dans le cadre de la loi de dé-communisation, pris le nom de KP en 2016.

7. C'est sur les concordances entre les témoignages que nous bâtissons un tableau de la formation, de la vie quotidienne des élèves africains à Perevalnoe. 
C'est l'étape indispensable pour aborder les spécificités de ces formations en même temps que les traits communs à nombre de systèmes de formation militaires.

Le Centre d'enseignement-165, Perevalnoe, fut le plus important pour la formation des spécialistes militaires des mouvements de libération nationale. Il constitue le point d'ancrage de cette première étape qui nous conduit aux spécificités de la formation linguistique, politico-idéologique et militaire des combattants des mouvements de libération africains, et nous invite à nous interroger sur le devenir des différents militaires africains formés en URSS.

De la formation des combattants de mouvements de libération à la formation des officiers

Créé en Crimée en 1965, conformément à la «Déclaration sur l'octroi de l'indépendance aux pays et peuples coloniaux » adoptée lors de l'Assemblée générale de l'onu en 1960, le Centre d'enseignement-165 Perevalnoe ${ }^{8}$, situé près du bourg du même nom à 21 kilomètres de la route SimferopolAlouchta, fut le premier à accueillir les combattants des mouvements de libération nationale et des partis africains : le Parti africain pour l'Indépendance de la Guinée-Bissau et les îles du Cap-Vert, PAIGC, l'Organisation du peuple du Sud-Ouest africain SWAPO (Namibie), l'Union du peuple africain du Zimbabwe (Rhodésie du Sud/Zimbabwe), le Mouvement populaire pour la libération de l'Angola, MPLA, le Front de libération du Mozambique, FRELIMO, le Congrès national africain, ANC.

Alors que Moscou restait évasif quant à son soutien militaire à ces mouvements de libération, V. G. Solodovnikov, directeur de l'Institut d'Afrique et chef de la délégation soviétique à la Conférence internationale d'appui aux peuples des colonies portugaises, réunie à Rome en juin 1970, admit dans une interview à la Pravda que l'uRss fournissait aux combattants pour la liberté dans ces pays « des armes, des moyens de transport et de transmission [...], indispensables pour une lutte victorieuse », et que les spécialistes militaires et civils membres des organisations de libération, étaient formés en URSS (Pravda, 7 juillet 1970, cité par V. Shubin 2013: 18).

De nombreux témoignages sont venus ensuite corroborer ces propos. L'un des interprètes ayant répondu à l'enquête de la Komsomolskä̈a Pravda explique :

Pendant deux ans (1974-1976), j'ai eu à servir sur ce site en tant qu'officier-interprète. [...] Je pense que tout cela n'est plus secret depuis longtemps et que je peux raconter ce que j'ai vu. [...] À partir de la deuxième moitié des années 1970, la presse soviétique [...] faisait de temps en temps mention d'affirmations «calomnieuses » de la presse bourgeoise racontant qu'en URSS, quelque part en Crimée, existe un

8. Le mot pereval en russe veut dire «franchissement» ou «dépassement » en français. 
camp secret où l'on prépare des terroristes internationaux. Ces déclarations, naturellement, étaient démenties avec indignation. Cependant, un tel établissement, sous le nom de Centre d'enseignement-165, a bel et bien existé jusqu'en 1990, pour préparer des militaires étrangers (Zubarev 2001 : s. p.).

Les interprètes et traducteurs qui y ont travaillé sont unanimes pour déclarer que le Centre d'enseignement-165 n'était en fait pas du tout secret, ni pour les services secrets occidentaux, ni pour les habitants du lieu dont un grand nombre (environ 500 salariés) entretenait le site. Ce site ne représentait un secret que pour les centaines de milliers de citoyens soviétiques qui se rendaient dans les stations balnéaires et les sanatoriums de Crimée. D'ailleurs, Yuri Gorbunov, traducteur d'espagnol et de portugais à Perevalnoe dans les années 1970, observe, photographies à l'appui, que malgré tout le secret qui entourait le Centre-165,

on voyait en Crimée nos élèves dans les rues, dans les écoles, les entreprises de Simféropol, on les accueillait aimablement dans les kolkhozes et les sovkhozes. On les invitait aux manifestations festives des travailleurs de Crimée et eux, depuis la tribune installée près du bâtiment du comité exécutif régional et du monument à Lénine, suivaient avec attention les colonnes festives?

À vrai dire, «ce qui restait secret concernait essentiellement les élèves : qui étaient-ils, combien, et de quel pays venaient-ils? Presque toutes les armes étaient non secrètes, et l'URSS en faisait ouvertement commerce. Nous n'avions aucune relation avec le KGB » (Zubarev 2001 : s. p.). Sur la question du renseignement et de la sécurité intérieure, Yuri Gorbunov précise : «Dans toutes les armées du monde, on trouve obligatoirement des gens du contre-renseignement dans les unités. Et là où apparaissent des militaires étrangers, les gens du KGB ont autre chose à faire que de "perlustrer la correspondance" des lieutenants $\gg{ }^{10}$.

De son côté, Dmitri Zubarev raconte que :

si dans les journaux apparaît un entrefilet annonçant que Sam Nujoma de Namibie, Joshua Nkomo de Rhodésie ou Agostinho Neto d'Angola est arrivé en URSS à l'invitation du Comité de solidarité avec les peuples d'Asie et d'Afrique, on peut être sûr qu'il mène des négociations sur la préparation de ses combattants, et que bientôt il nous faudra attendre l'arrivée du contingent correspondant (Zubarev 2001 : s. p.).

Il explique comment les rangs d'élèves africains s'agrandissaient à Perevalnoe :

9. Les souvenirs de Yuri Gorbunov ont pour titre Крым партизаны для Африки (Crimée, Les partisans pour l'Afrique) et sont publiés sur <http://topwar.ru/ 37349-krym-partizany-dlya-afriki-chast-1.html>.

10. <http://topwar.ru/37349-krym-partizany-dlya-afriki-chast-2.html>. 
Tard dans la nuit [...] atterrissait sur l'aéroport de Simferopol un avion soviétique qui ne figurait pas sur l'horaire. Il arrivait de quelque pays africain ami ou d'un pays arabe ayant avec nous une frontière commune, où il y avait la guerre [...]. À bord de l'avion se trouvait un groupe d'élèves non armés, dans les tenues les plus diverses, avec à leur tête un responsable politique du groupe. Il suivait lui aussi la même formation. L'âge moyen de ceux qui arrivaient était entre 15 et 30 ans ${ }^{11}$. Un officier soviétique accompagnait le groupe. [...] Près de l'échelle, des autobus avec les stores baissés, qui appartenaient aux unités. On faisait monter ceux qui venaient d'arriver dans les autobus et une demi-heure plus tard ils étaient à Perevalnoe [...] (Zubarev 2001 : s. p.).

\section{Evgueni Loguinov rapporte que :}

La première promotion du Centre-165 fut constituée en mars 1966. C'était des partisans de Guinée-Bissau. Ils se battaient alors contre les colonisateurs portugais, sous les drapeaux du PAIGC. Parmi les diplômés de Crimée s'est particulièrement distingué sur les fronts guinéens José Marquez: il fut nommé chef du département stratégique de l'état-major général, et le gars discret surnommé Brown devint un héros national $^{12}$. [...] D'autres Guinéens arrivèrent pour les remplacer et aussi encore 200 combattants d'Angola et du Mozambique. De tous ceux-ci, c'est surtout Ribeira qui a eu de l'avancement ${ }^{13}$.

Les leaders des mouvements africains de libération nationale, parmi lesquels Agostinho Neto, Amilcar Cabral, Sam Nujoma ${ }^{14}$, Oliver Tambo ${ }^{15}$ furent invités au Centre. «Tous, lors des réunions devant les officiers, exprimaient leur satisfaction du haut niveau de préparation des élèves. Ils comprenaient à quel point c'était difficile d'instruire des partisans illettrés [...], ne parlant que les langues tribales $[\ldots] \gg{ }^{16}$.

Dans les années 1960-1970, le Centre-165 formait essentiellement des partisans, des combattants et des commandants pour les armées révolutionnaires de libération nationale qui se battaient pour l'indépendance en Angola, au Mozambique, au Congo, au Zimbabwe, ou en Guinée ; il y avait 300 à 400 personnes en formation en même temps. De mieux en mieux aménagée,

11. «En tout, les insurgés d'Amilcar Cabral étaient 75, ayant entre 16 et 35 ans », voir Centre de préparation de terroristes (<http://feldgrau.info/other/10903-tsentrpodgotovki-terroristov $>$ ), publié le 6 novembre 2014.

12. E. Loguinov, 2007, СВОУ (Симферопольское военное объединенное училище или 165 Учебный центр подготовке иностранных военнослужащих). Перевальное в CCCP и сегодня. (SVOU. L’École militaire unifiée de Simferopol ou Centre de préparation des militaires étrangers, 165, Perevalnoe en URSS et aujourd'hui), <http://vkimo.com/node/2012>.

13. Revenu au Mozambique, son pays, Ribeira prit peu après la tête de la Direction politique principale de l'armée d'insurrection. Bientôt, il passa dans l'opposition, devint un de ses leaders, et «tourna le canon de son fusil contre ses anciens camarades de réfectoire de Crimée » (Lvovski 2005, s. p.).

14. Le fils aîné de Sam Nujoma étudiait à Perevalnoe pour devenir aide-médecin militaire.

15. Vice-président puis président en exil de l'ANC.

16. <http://topwar.ru/37349-krym-partizany-dlya-afriki-chast-1.html>. 
la base de Perevalnoe fut agrandie et deux ans environ après son ouverture, elle pouvait accueillir jusqu'à 500 combattants pour des cours de six à huit mois.

Mais, en règle générale [...], il n'y avait habituellement pas plus de 300-400 élèves en même temps, de sorte qu'en moyenne 1000 personnes par an étaient formées. On dit qu'en dix ans, toute l'armée de partisans de Guinée-Bissau a pu y améliorer sa qualification, et que certains combattants sont revenus plusieurs fois [...]. Pendant les 10 premières années d'existence du centre, les combattants des colonies anglophones et lusophones d'Afrique représentaient l'écrasante majorité des élèves ${ }^{17}$.

En 1980, Perevalnoe a été transformé en École militaire unifiée de Simferopol ${ }^{18}$, faisant disparaître par la même occasion, selon Evgueni Loguinov, les «disciplines pour partisans » du programme. À la différence du centre d'enseignement, la formation dans l'École désormais dispensée en russe pour une durée de deux ans ne préparait plus des partisans, mais des officiers cadres; les élèves recevaient le grade de lieutenant dans leur armée nationale à la fin de leurs études. Parallèlement, étaient dispensés des « cours d'amélioration de la qualification » de courte durée ${ }^{19}$. À la fin de ces cours, les promotions comptaient des commandants, artilleurs, sapeursmineurs, officiers de transmission, opérateurs d'artillerie pour la défense aérienne, techniciens pour l'armement, voire des infirmières. Car à Perevalnoe étudiaient aussi des femmes ${ }^{20}$.

Au cours des douze premières années d'existence du Centre, des milliers d'élèves d'Éthiopie, de Guinée-Conakry, de Madagascar, du Mali, de Zambie, de Tanzanie, du Congo, du Bénin, de Sao Tomé-et-Principe y ont suivi une préparation. Les meilleurs d'entre eux ont, par la suite, servi jusqu'aux galons de général dans les armées de leurs pays ${ }^{21}$. Jusqu'à la chute de l'URSs, pas moins de 15000 «spécialistes ayant un large profil » ${ }^{22}$ ont été diplômés du centre de Perevalnoe ${ }^{23}$.

17. «Курсанты для борьбы за социализм во всем мире («Étudiants dans la lutte pour le socialisme dans le monde entier »), <http://index.org.ru/journal/ 19/zubar19.html>.

18. L'École militaire unifiée de Simferopol cessa d'exister en 1992, cédant la place à la $84^{\mathrm{e}}$ Brigade mécanisée du $32^{\mathrm{e}}$ Corps d'armée des Forces armées ukrainiennes.

19. <http://vkimo.com/node/2012>.

20. Nous n'avons jusqu'à présent pas trouvé dans les différentes sources consultées de témoignage de l'une d'entre elles.

21. <www.segodnya.ua/oldarchive/c2256713004f33f5c2256fea00516140.html>.

22. E. Loguinov donne un chiffre encore plus important : 18000 combattants pour les divers mouvements de libération nationale dans les pays d'Asie, d'Afrique, du Proche-Orient, <http://vkimo.com/node/2012>.

23. <http://www.vko.ru/biblioteka/na-afrikanskom-kontinente>. 


\section{Apprentissage de la langue russe}

La question de l'apprentissage du russe par les étudiants africains, civils et militaires, devint rapidement une préoccupation importante pour les autorités soviétiques. Des cours préparatoires pour étudier le russe et les fondements de la préparation militaire n'étaient cependant pas envisageables au début pour les combattants africains de mouvements de libération. Il eut fallu en effet recruter un corps de traducteurs suffisamment dense, et que le matériel didactique fût prêt, et traduit dans les langues étrangères ${ }^{24}$. Des facultés préparatoires destinées aux militaires étrangers virent le jour, à partir de janvier 1950, dans lesquelles étaient créées des chaires de russe, langue étrangère ; une préparation militaire spéciale y était réalisée en même temps que les militaires étrangers étudiaient le russe (Bulkov 2007 : 44 ; Romashov 2010 : 44 ; Tkachev 2014 : 1117). Huit à dix mois (ultérieurement dix à onze mois) constituaient le temps jugé nécessaire pour acquérir la langue ${ }^{25}$.

Cependant, ces instructions ne s'appliquèrent que peu aux contingents africains. Il n'était en effet guère possible de réaliser ce type de préparation lorsque les élèves concernés étaient «pratiquement illettrés » et ne parlaient souvent pas la langue des anciennes métropoles ${ }^{26}$. Yuri Gorbunov souligne à ce propos qu'à Perevalnoe : «Au début, avec les partisans [Bissau] guinéens, le traducteur n'arrivait même pas à s'expliquer. Il s'avéra que les gars à peau sombre ${ }^{27}$ parlaient différents dialectes, et que très peu d'entre eux parlaient le portugais, et encore très superficiellement... $»^{28}$. Ce que confirme Mickael Lvovski (2005 : s. p.) : «Beaucoup de combattants venus d'Afrique, ne parlaient pas les langues européennes. Dans un même groupe il y avait souvent des représentants de trois à cinq tribus qui ne se comprenaient pas entre eux.»

Lors des premières étapes de la formation des élèves africains, une charge considérable reposait de fait sur les traducteurs. Faute de temps pour étudier le russe, les Africains étudiaient les autres matières avec l'aide des

24. Au début, ces études n'étaient pas gratuites. Mais très vite des accords pour la gratuité de la formation furent conclus avec de nombreux pays en voie de développement, dont des pays africains. Pour l'URSS cela contribuait sans doute à avoir plus d'autorité, influait positivement sur la réalisation des intérêts géopolitiques. Bien que dans les dernières années de l'URSS l'enseignement sur la base de la gratuité ait eu certains résultats négatifs, la préparation des militaires étrangers, notamment de ceux des pays comme l'Algérie, la Libye, fut une source d'entrée de devises importante (DIRECTION GÉNÉRALE DE LA COOPÉRATION MILITAIRE INTERNATIONALE $2001:$ 124).

25. Après la faculté préparatoire, l'étude de la langue russe se poursuivait au sein de groupes réunissant trois à cinq élèves selon leur appartenance nationale et leur niveau d'instruction (TSEPIN \& CHERNIAIKOV 2014 : 66).

26. Nous parlons ici des combattants des mouvements de libération dont un certain nombre provenant des zones rurales n'avaient jamais été scolarisés.

27. Comparativement aux combattants «métis».

28. <http://topwar.ru/37349-krym-partizany-dlya-afriki-chast-1.html>. 
traducteurs qui connaissaient l'une des langues européennes, car les enseignants ne savaient que le russe.

\section{Formation politique}

Dans le programme d'enseignement pour les combattants de libération, du temps était réservé à la préparation politique, ce qui s'accordait avec l'idée d'éveiller chez les élèves un esprit d'égalité et de justice, et la certitude de la justesse de la cause qu'ils servaient. Il s'agissait notamment de familiariser les Africains avec la théorie du marxisme-léninisme et avec l'histoire des mouvements révolutionnaires mondiaux. Aussi, les traducteurs ne devaient pas seulement traduire la terminologie militaire mais préparer idéologiquement les nouvelles recrues africaines en leur enseignant les rudiments du marxisme-léninisme. La difficulté ne résidait alors pas tant dans la connaissance de la terminologie politique que dans la capacité à rendre des idées politiques complexes compréhensibles pour des élèves ayant fait peu d'études. Mais l'essentiel, comme l'enseignait à Perevalnoe le colonel Antipov ${ }^{29}$, «était de leur apprendre d'abord sur qui et pourquoi tirer, et seulement après de leur enseigner comment tirer et utiliser les explosifs [...] $»^{30}$.

Cette préparation politique était obligatoire ${ }^{31}$, mais elle n'occupait de fait pas beaucoup de temps et était ajustée pour chaque contingent d'élèves. «Pendant ces années-là, on étudiait l'histoire des mouvements révolutionnaires mondiaux — de Marx jusqu'à la dite troisième étape du mouvement révolutionnaire mondial que les étudiants étaient d'ailleurs en train de vivre. » Pour les élèves, tout cela était plutôt compliqué : «c'est qu'ils n'avaient jamais vu une carte de géographie...» (Zubarev 2001 : s. p.). Dans certains groupes d'Afrique lusophone, comme certains ne comprenaient pas le portugais, donc :

Il fallait désigner, parmi les gens qui maîtrisaient le portugais et les dialectes tribaux, des traducteurs intermédiaires. C'est-à-dire qu' on utilisait « une double traduction ». Le traducteur soviétique traduisait en portugais, et le traducteur intermédiaire africain disait ce qu'il avait compris dans le dialecte tribal. Souvent le traducteur soviétique et les traducteurs intermédiaires étaient obligés d'expliquer aux élèves les avantages du socialisme, plus longuement que le professeur ${ }^{32}$.

«Que restait-il de la théorie après la double traduction, on n'en sait rien, d'autant que les élèves ne pouvaient pas prendre de notes » (Lvovski 2005 :

29. Le colonel A. I. Antipov, chef du cycle socio-politique du Centre d'enseignement165 , était l'un des officiers les plus respectés du centre, <http://topwar.ru/37349krym-partizany-dlya-afriki-chast-1.html $>$.

30. <http://topwar.ru/37349-krym-partizany-dlya-afriki-chast-1.html>.

31. Dans les universités et établissements d'enseignement pour civils, la formation idéologique n'est devenue obligatoire qu'en 1968 (KATSAKIORIS 2015 : 206).

32. <http://topwar.ru/37349-krym-partizany-dlya-afriki-chast-1.html>. 
s. p.). Dans ce contexte, les tensions ne manquaient pas, comme en témoignent certains traducteurs, à propos d'un enseignement idéologique qui pouvait générer des désaccords importants entre les responsables politiques accompagnant les militaires africains et l'encadrement soviétique, notamment à propos de la Chine :

Un des thèmes importants était alors chez nous [en URSS] la lutte contre le maoïsme, or il y avait chez nous des élèves venant du Mozambique. Quand leur responsable politique comprit que, sous l'apparence de préparation politique était menée une propagande antichinoise, il posa catégoriquement la question à son commandement, à savoir s'il fallait arrêter ces cours. Et il fallut leur faire des concessions (Zubarev 2001 : s. p.).

\section{Formation militaire}

Lors de la préparation militaire des combattants de libération, qui durait de six à dix mois, les particularités géographiques des pays d'où venaient les élèves étaient prises en considération : des opérations de formation étaient organisées dans les montagnes, en forêt, en mer ou sur des fleuves. Les élèves apprenaient à fabriquer et à utiliser les explosifs, s'emparer de dépôts d'armes, organiser des diversions sur les centrales électriques et les sites militaires, prendre d'assaut des bâtiments et faire sauter des voies ferrées, réaliser des raids sur les commissariats de police, enlever des sentinelles, tirer au pistolet, à l'arme automatique, à la mitrailleuse, jeter des grenades. Simultanément, ils étudiaient activement la tactique et les règlements militaires des armées coloniales.

Dans ses souvenirs, Yuri Gorbunov note que l'enseignement donné aux Africains comportait l'apprentissage du travail de diversion sur les sites gardés par les armées coloniales; pour leur permettre d'acquérir les subtilités nécessaires à la conduite de la guérilla et de certaines opérations spécifiques, des jeux de rôle étaient organisés ${ }^{33}$. Des dispositifs d'artillerie spéciaux avaient été élaborés pour les partisans, qu'on ne trouvait pas dans l'armement de l'armée soviétique, par exemple une installation de défense antiaérienne pouvant être chargée sur le dos et se montant en deux minutes.

Dans le même esprit, à partir d'un lance-roquettes multiple appelé Katioucha, les constructeurs soviétiques avaient fabriqué un mortier à un seul canon qui pouvait être porté par deux hommes. Cependant, de façon générale, on apprenait aux partisans à utiliser les armes en usage :

Nos élèves étaient fréquemment complètement illettrés, et néanmoins on arrivait à les former avec succès. Par exemple, pour effectuer un calcul très simple pour faire sauter un pont, il fallait connaître l'arithmétique jusqu'à 1000 ; et nous leur enseignions les chiffres et les opérations arithmétiques selon une méthode spéciale pour illettrés. Un jeune Africain normal est un matériau suffisamment malléable,

33. <http://topwar.ru/37349-krym-partizany-dlya-afriki-chast-1.html>. 
dont on peut faire un bon soldat. [...] La plupart des élèves au cours de leurs études atteignaient dans leur spécialité le niveau de sergent de l'époque de la Grande Guerre patriotique ${ }^{34}$.

De toute évidence, une forme de fierté du travail accompli transparaît dans ces témoignages d'anciens enseignants et traducteurs qu'accompagne souvent un sentiment de supériorité à l'égard de leurs élèves africains, parfois teinté de commisération ou d'irritation ${ }^{35}$.

\section{La vie dans le centre et à l'extérieur}

À leur arrivée à Perevalnoe, comme d'ailleurs dans d'autres centres de formation $^{36}$, les armes et l'alcool que les élèves avaient apportés leur étaient retirés. Ils étaient ensuite répartis en chambrées de dix, chacune regroupant préférentiellement des élèves de même origine ethnique. La discipline était rigoureuse : au début de chaque semaine, dès le matin, tout le personnel du centre d'enseignement - les officiers enseignants dans les différents cycles, la compagnie de la garde, l'état-major et quatre compagnies d'élèves africains - se mettait en rangs sur la place d'armes. Le commandant fixait les tâches à accomplir durant la semaine, informait sur les infractions à la discipline, et exprimait sa reconnaissance. Ensuite tous défilaient, au son d'un orchestre d'instruments à vent, devant la tribune sur laquelle se trouvait la direction de l'unité : le commandant, son adjoint, le chef du département politique.

Pour la durée de leur séjour, un ensemble de vêtements civils leur était fourni. Ils recevaient en outre quelques roubles chaque mois comme argent de poche.

L'entretien d'un élève coûtait de 7 à 9000 roubles/an à l'URSS. Entre autres, cela incluait la solde de 20 roubles par mois et par personne, les vêtements civils : costume composé d'un veston et d'un pantalon, une chemise, une cravate, un chapeau, des chaussures et un imperméable, le tout représentant une somme de 260 roubles. Les élèves étaient nourris avec des plats de la cuisine russe, non pas comme l'étaient les soldats soviétiques, à 90 kopeks par jour, mais selon la norme dite d'été, revenant à 3 roubles 50 kopeks. La Libye, l'Éthiopie (ainsi que le Laos) étaient les seuls pays qui payaient pour la formation de leurs citoyens en argent «réel». Tous les autres étudiaient l'art de la guerre à crédit ${ }^{37}$.

Certains problèmes étaient liés aux difficultés d'assimilation d'une autre culture du quotidien, aux relations avec les élèves soviétiques, ou encore à

34. <http://index.org.ru/journal/19/zubar19.html>.

35. Celle-ci s'expliquait parfois par un désir de transmettre rapidement les connaissances.

36. Les futurs élèves venant d'Afrique étaient d'abord mis en quarantaine durant une semaine pour vérifier qu'ils n'étaient pas porteurs de maladies transmissibles graves (lèpre, gale, syphilis, etc.), car de tels cas avaient été enregistrés au tout début.

37. <http://topwar.ru/37349-krym-partizany-dlya-afriki-chast-1.html>. 
la nourriture (les Guinéens, par exemple, ne mangeaient pas de bouillie de sarrasin, pensant que c'était du « riz avarié »). Pourtant, les rations destinées aux élèves étaient les plus riches parmi celles que proposait l'armée soviétique ; ils avaient droit à la « ration renforcée d'été », à savoir la catégorie supérieure de l'approvisionnement de l'armée, rarement appliquée.

En gros, les élèves étaient nourris au niveau d'une bonne maison de repos de syndicat soviétique [...]. Le menu se différenciait tellement du menu habituel du soldat qu'il était interdit aux soldats servant dans les unités de s'approcher du bâtiment où se trouvait la cantine des élèves [...] (Zubarev 2001 : s. p.).

Le rythme des activités était soutenu et les exercices avaient lieu dans un climat assez tendu : lever à six heures, gymnastique, petit-déjeuner, déjeuner et dîner - toujours en ordre de formation. Entre le petit-déjeuner et le déjeuner, cinq heures d'exercices. Après le déjeuner, travail personnel ou participation à l'aménagement du territoire du centre et des sites de formation. « À partir de $18 \mathrm{~h}$, on leur projetait au club des films soviétiques sur la guerre et la révolution. [...] Après le dîner, à partir de $21 \mathrm{~h}$ les élèves allaient faire les exercices de nuit conformes à l'horaire avec les enseignants et les traducteurs $»^{38}$. Les exercices nocturnes étaient nombreux : par exemple, pour la préparation «génie-diversion» on installait des mines, les sapeurs faisaient des passages dans les champs de mines. Soumis à ce régime, les élèves grognaient parfois et certains disaient que la discipline était «comme en Chine » (Zubarev 2001 : s. p.).

Organisée sur un strict modèle militaire, surchargée d'exercices et d'efforts physiques, la vie dans le Centre-165 ne permettait guère aux élèves de se relâcher. Dans l'ensemble, les manquements à la discipline étaient rares ; on signalait parfois des bagarres entre élèves, et aussi des tentatives de franchissement de l'enceinte pour avoir des contacts avec des femmes (ibid.).

Sortir des limites du centre n'était autorisé que dans le cadre de visites dans des institutions soviétiques modèles, au titre de la formation politique ; celles-ci étaient organisées environ une fois par mois dans les kolkhozes, les fabriques, les écoles, les magasins de Simferopol, où « [...] ils [les élèves] pouvaient se convaincre de leurs propres yeux des avantages du socialisme soviétique. Ils voyaient en Crimée des kolkhozes riches et prospères, des stations balnéaires populaires sur la côte sud pour les travailleurs, les entreprises de Simferopol $»^{39}$.

Il faudrait prolonger la description et analyser les multiples incidents relatés par les traducteurs et les interprètes afin de mettre au jour les espoirs, les tensions et les désillusions des acteurs. Les témoignages rapportés ici et la description réalisée ont pour seule ambition de proposer un premier aperçu sur la formation et la vie quotidienne des militaires, notamment des combattants des mouvements de libération à Perevalnoe et de susciter des

38. <http://topwar.ru/37349-krym-partizany-dlya-afriki-chast-1.html>.

39. Ibid. 
recherches. On voudrait notamment connaître le point de vue des élèves africains du Centre-165 qui parfois cependant se laisse deviner à travers l'évocation de leur cursus, emploi du temps et de leurs conditions de vie; quant à leur devenir, il n'est quasiment pas évoqué dans les sources consultées.

\section{Le devenir des militaires formés en URSS}

En l'absence d'enquête spécifique auprès d'anciens partisans ou officiers passés par le Centre-165, quelques données sur le devenir des anciens militaires formés en URSS permettent de découvrir une situation assez contrastée en ce qui concerne leur vie professionnelle après le passage en URSS dans les établissements militaires et après les guerres. Trois cas assez différents en témoignent : alors qu'en Angola, ceux-ci ont obtenu une certaine reconnaissance, la défiance reste toujours grande au Mozambique, tandis qu'en Éthiopie, ils sont devenus quasiment invisibles.

En Angola, nombre de diplômés des établissements d'enseignement militaire soviétiques ont occupé ou occupent parfois encore aujourd'hui des fonctions élevées dans les structures dirigeantes du pays ; le nom du général Francisco Lopes Gonçalves Afonso « Hanga », son nom de guerre, est souvent cité $^{40}$. Au $1^{\text {er }}$ janvier 1995, de nombreux militaires angolais avaient étudié dans les établissements militaires soviétiques (Shubin 2006), plusieurs d'entre eux à Perevalnoe; d'autres passèrent par une école de guerre en Bulgarie, en Tchécoslovaquie ou en RDA, voire en Algérie. Selon un «inventaire » des diplômés des établissements d'enseignement militaire soviétiques, réalisé en 1989 par les services diplomatiques d'URSS à Lobito dans la province de Benguela, la plupart des 106 personnes retrouvées, dont 94 étaient des anciens officiers des Forces armées populaires de l'Angola (FAPLA) occupaient alors des postes de direction dans l'appareil du Parti et de l'administration de la province ou dans l'armée.

Dans la République populaire du Mozambique, le consulat général d'URSS à Beira a aussi fait des recherches en 1987 qui ont permis de retrouver près d'une centaine de Mozambicains ayant terminé leurs études dans des écoles supérieures ou secondaires spécialisées, y compris militaires. Cela fut difficile, notent les rapports diplomatiques, car nombre d'entre eux ne voulaient pas dire qu'ils avaient fait des études en URSS, évitaient les contacts avec les Soviétiques pour ne pas attirer sur eux l'attention de leur employeur ${ }^{41}$. D'ailleurs, selon les diplômés, les chefs mettaient des bâtons dans les roues à ceux ayant étudié en URSS. D'une part, ils devaient à leur retour effectuer

40. Chef d'état-major des Forces aériennes et personnalité légendaire dans les forces armées angolaises, sorti d'une école d'aviation d'URSS, «Hanga » a dirigé pendant vingt ans les forces militaires aériennes de l'Angola.

41. Information du Consulat général d'URSS à Beira, République populaire du Mozambique «Travail du Consulat général avec les Mozambicains diplômés des établissements soviétiques », 29 février 1988. 
un stage de perfectionnement de 45 jours avant même d'aborder la question de leur affectation à un poste. D'autre part, très souvent, les diplômés attendaient encore plusieurs mois, voire plus longtemps, avant d'obtenir le grade d'officier et l'on ne leur proposait que rarement un poste correspondant à leur spécialisation.

L'Éthiopie propose l'un des exemples les plus éclatants de la « dévaluation » des contingents militaires formés en URSS, due notamment à la chute du régime de Mengistu Hailé Mariam et à l'arrivée au pouvoir de nouvelles forces politiques. Au $1^{\mathrm{er}}$ janvier 1995, le nombre d'Éthiopiens ayant terminé leur formation militaire en URSS/Russie atteignait $5794^{42}$. Quelles ont été leurs destinées ? S. V. Mezentsev, de l'Institut d'Afrique de Moscou, évoque l'ampleur du déclassement, voire de l'éviction :

[...] On arrive dans la capitale éthiopienne, à l'hôtel Hilton. Qui est-ce qui lève la barrière à l'entrée ? Des colonels, des lieutenants colonels qui parlent le russe, qui ont étudié chez nous (en URSS) [...]. Notre coopération militaire active a commencé en 1977 et s'est achevée en 1991 [...]. Les colonels qui lèvent les barrières à l'entrée des hôtels, ce n'est pas le résultat du chômage; ce sont des militaires de l'armée de Mengistu, qui combattaient ceux qui sont maintenant arrivés au pouvoir [...]. En 1991, lorsqu'ils (le Front démocratique révolutionnaire des peuples éthiopiens) ont pris Addis-Abeba, ils les ont tous envoyés dans des camps de filtration comme ennemis du nouveau pouvoir [...]. Ils se sont retrouvés dans des camps, qui pour un an, qui pour six mois. Certains [...] sont passés du côté du nouveau pouvoir, et alors ils (les chefs du nouveau pouvoir) les ont pris comme adjoints [...] et ils les ont utilisés pendant quelque temps dans les forces armées, tant qu'ils n'avaient pas appris à faire les choses eux-mêmes. Et ensuite ils se sont débarrassés d'eux. Ils les ont utilisés dans les années 1991-1995 [...]. Concernant le personnel de commandement - ceux qui avaient participé aux actions armées - certains furent fusillés, d'autres incarcérés pour longtemps, certains simplement écartés. La plupart des spécialistes de haut niveau - jusqu'à $90 \%$ de ceux préparés en URSS - ne furent pas rappelés à leur poste [...] (Entretien, Institut d'Afrique de l'Académie des sciences de Russie, Moscou, 14 juillet 2016).

\section{Les associations de diplômés d'écoles soviétiques}

Que reste-t-il, pour ces nombreuses élites militaires africaines de leur séjour en URSS ? Si plusieurs d'entre elles ont occupé et occupent parfois encore aujourd'hui des postes de direction dans l'appareil militaire de leur pays, par exemple en Angola, elles ne revendiquent pas leur formation soviétique, sauf dans des pays où existent des regroupements d'anciens diplômés militaires et civils des établissements soviétiques. Or, les tentatives de regroupement des diplômés des écoles soviétiques ou russes, y compris militaires,

42. <www/vko.ru/sites/all/themes/vpk/templates/node-article.tpl.php>. 
n'ont guère abouti en Afrique subsaharienne et n'aboutissent toujours pas aujourd'hui. L'Association congolaise des anciens étudiants des universités des Républiques de l'ex-URSS pour le développement et l'entraide, créée en 2000, est l'une des très rares unions professionnelles en Afrique subsaharienne. Dans les autres pays, la création d'unions indépendantes et l'intégration des militaires formés en URSS dans des associations de diplômés d'établissements soviétiques ou de pays socialistes ne progressent pas. Ainsi, alors qu'il existe en Russie depuis 2004 une Union des vétérans d'Angola, les tentatives pour regrouper les diplômés angolais, civils et militaires, d'URSS, entreprises à plusieurs reprises en Angola, n'ont abouti à aucun résultat ${ }^{43}$. Il faut croire que la chute du mur de Berlin et l'éclatement de l'Union soviétique ont révélé, à côté de la marginalisation des idéaux socialistes dans ces pays, la réalité de leur formation sociale avec ses divisions ethniques, nationales ou professionnelles, privilégiant le retour dans le giron des anciennes puissances coloniales dont les diplômes se trouvent revalorisés pour l'occasion.

Institut d'Afrique, Académie des Sciences de Russie, Moscou.

Traduit du russe par Sonia Colpart.

\section{BIBLIOGRAPHIE}

Alexander, J. \& McGregor, J. A.

2017 «African Soldiers in the USSR : Oral Histories of ZAPU Intelligence Cadres' Soviet Training, 1964-1979 », Journal of Southern African Studies, 43 (1) : 49-66.

Bulkov, A. A.

2007 «Историко-педагогический анализ развития процесса профессиональной подготовки иностранных военнослужащих в российских военных вузах » (Analyse historico-pédagogique du développement du processus de préparation professionnelle des militaires étrangers dans les établissements d'enseignement supérieur russes), Право и образование (Droit et formation), $2: 49-53$.

DIRECTION GÉNÉRALE DE LA COOPÉRATION MILITAIRE INTERNATIONALE

2001 Главное Управление международного военного сотрудничества Министерства обороны Российской Федерации (1951-2001). Исторический очерк (Direction générale de la coopération militaire internationale du ministère de la Défense de la Fédération de Russie (1951-2001). Essai historique). Москва, Главное Управление международного военного соторудничества МО РФ, Институт военной истории МО РФ (Moscou, Direction générale de la coopération militaire internationale, Ministère de la Défense de la Fédération de Russie).

43. Journal du directeur du Département consulaire de l'Ambassade d'URSS en Angola, 20 avril 1989. 
KATSAKIORIS, C.

2015 La formation des étudiants africains et arabes en URSS pendant la guerre froide, Thèse de doctorat, Paris, EHESS.

Korendiasov, E. N.

2015 «Военно-техническое сотрудничество России с Африкой » (La collaboration militaire et technique de la Russie avec l'Afrique), Азия и Африка сегодня (L'Asie et l'Afrique aujourd'hui), 9 : 9-18.

Lvovski, M.

2005 «Школа террористов с марксистским уклоном : 40 лет назад в Крыму появился Центр подготовки бойцов и командиров для повстанческих армий стран Азии и Африки (L'école des terroristes à tendance marxiste : il y a 40 ans en Crimée a été créé le Centre de préparation de combattants et commandants pour les armées d'insurrection des pays d'Asie et d'Afrique), Сегодня : Ежедневная общественно-политическая газета (Segodnia, Quotidien socio-politique), Киев : ЗАО « Издательская группа „Сегодня” » (« Groupe éditorial Segodnia »), Kiev, no 90 (2032), 22 avril, $<$ www.segodnya.ua/oldarchive/c2256713004f33f5c2256fea00516140>.

Mazov, S. V.

1999 «Африканские студенты в Москве в Год Африки (Les étudiants africains à Moscou [1'année de l'Afrique]), Vostok, 3 : 91-93.

Romachov, V. M.

2010 «Оптимизация обучения иностранных военнослужащих основам само-стоятельной работы в военных вузах России » (Optimiser l'enseignement des bases du travail indépendant dans les établissements d'enseignement supérieur militaires de Russie), Вестник Военного университета (Le Messager de l'université militaire), 21 (1) : 42-45.

SHAIKIN, V. I.

2013 Братья по оружию. Подготовка иностранных военнослужащих. Исторический очерк (Frères d'armes. La préparation des militaires étrangers. Essai historique), Рязань, Научное издание (Riazan, Édition scientifique).

Shubin, V. G.

2006 «Beyond the Fairy Tales : The Reality of Soviet Involvement in the Liberation of Southern Africa », in M. Matusevich (ed.), Africa in Russia. Russia in Africa. Three Centuries of Encounters, Trenton, N. J., Asmara, Africa World Press Inc. : 331-352.

2013 Горячая «холодная война»: Юг Африки (1960-1990 22.) (Brûlante « Guerre froide » : le Sud de l'Afrique [1960-1990]), Москва, ЯСК (Moscou, JASK).

TKACHEV, V. A.

2014 «Сущность и содержание профессиональной подготовки иностранных военнослужащих в инженерных вузах МО РФ » (Essence et contenu de la formation professionnelle des militaires étrangers dans les établissements d'enseignement supérieur du Ministère de la Défense de la Fédération de Russie), Молодой ученьий (Jeune chercheur), 4 : 1116-1118. 
Tsepin, M. O. \& Cherniaikov, M. N.

2014 «Историко-педагогический анализ отечественного и зарубежного опыта тактико-специальной подготовки иностранных военнослужащих в военных инженерных вузах России » (Analyse historicopédagogique de l'expérience de la préparation pour ingénieurs «spécialité tactique » dans les établissements d'enseignement supérieur en Russie et à l'étranger), Мир образования - Образование в мире, Научно-методический журнал (Le Monde de l'Éducation - La Formation dans le monde, Revue de méthode scientifique), 1 (53).

ZuBAREv, D.

2001 «Arabskikh terroristov gotovili v Krymu» (Des terroristes arabes étaient formés en Crimée), Komsomolskaïa Pravda, 28 sept. <http://index.org.ru/ journal/19/zubar19.html>.

\section{RÉSUMÉ}

Ouvert en Crimée en 1965, le Centre d'enseignement-165, Perevalnoe, fut le premier à accueillir pour des formations de courte durée les combattants des mouvements de libération nationale ; en 1980, il fut transformé en École militaire unifiée de Simferopol, préparant des officiers cadres. Cet article donne la parole aux traducteurs, enseignants russes de ce centre, longtemps tenu "secret » dont les témoignages, apparus sur les sites et forums de vétérans et dans la Komsomolskaïa Pravda, constituent une source utile pour cette recherche exploratoire. Si la formation militaire n'a guère souffert de contestation, I'apprentissage de la langue ou de l'enseignement idéologico-politique a parfois entraîné des tensions entre encadrement soviétique et militaires africains.

\section{ABSTRACT}

The Perevalnoe Center and Military Training in the Soviet Union. - Opened in Crimea in 1965, the Perevalnoe Training Center-165, was the first to welcome national freedom fighters for short-term training. In 1980, it was transformed into a school to prepare high-ranking officers and renamed the Unified Simferopol Higher Military School. This article allows translators and Russian language teachers who worked at the Center a chance to speak, given that this longtime "secret" establishment has recently been discussed by eyewitnesses whose testimony given online on websites, such as veterans' forums, and in the newspaper Komsomolskaïa Pravda, constitute the sources for this initial research. If military training was hardly ever contested as such, language learning and ideological and political indoctrination sometimes caused tensions between the Soviet administration and African military trainees.

Mots-clés/Keywords: URSS, formation, militaires, mouvements de libération/USSR, training, education, military, liberation movements. 